\title{
The Efficacy and Safety of a Promising Single-Channel Endoscopic Closure Technique for Endoscopic Treatment-Related Artificial Ulcers: A Pilot Study
}

\author{
Yosuke Minoda $^{a}$ Eikichi Ihara $^{a}$ Haruei Ogino ${ }^{a}$ Keishi Komoria \\ Yoshihiro Otsuka $^{a} \quad$ Hiroko Ikeda $^{a}$ Mitsuru Esaki $^{a}$ Takatoshi Chinen $^{a}$ \\ Takahiro Matsuguchi $^{b}$ Shunsuke Takahashic Noriko Shigad \\ Rie Yoshimura ${ }^{d}$ Yoshihiro Ogawa ${ }^{a}$

\begin{abstract}
aDepartment of Medicine and Bioregulatory Science, Graduate School of Medical Sciences,

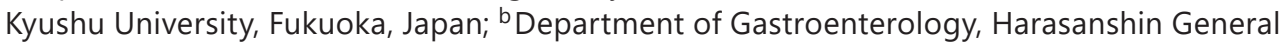
Hospital, Fukuoka, Japan; ' ${ }^{\circ}$ epartment of Internal Medicine, Fukuoka City Hospital, Fukuoka, Japan; ${ }^{d}$ Medical Treatment Corporate Foundation Group Hakuaikai Medical
\end{abstract} \\ Checkup Center Wellness, Fukuoka, Japan
}

\section{Keywords}

Single-channel endoscopic closure technique - Complete closure · Artificial mucosal ulcers . Endoloop

\begin{abstract}
Background/Aims: It is important to appropriately manage patients with procedure-related artificial mucosal ulcers or procedure-related complications. Many endoscopic closure techniques have been reported; however, they often require the use of special devices. We developed a single-channel endoscopic closure technique (SCCT) that can be performed with conventional devices. In the present study, we describe the technique and evaluate its efficacy. Methods: Twenty-five consecutive patients who underwent endoscopic treatment and whose artificial ulcer was closed using the SCCT were enrolled in this study. The technical success rate, number of clips for closure, procedure time, complication rate on the day of the procedure, clinical success rates on days 1 and 5 , and incidence of severe stenosis of the gastrointestinal (GI) tract at 2 months after the procedure were evaluated. Results: The median ulcer diameter was $20 \mathrm{~mm}$. The tumor locations were the stomach $(n=19)$, jejunum $(n=1)$, and colon $(n=5)$. The technical success rate was $100 \%(25 / 25)$, and the rate of incomplete closure was $0 \%(0 / 25)$. Eight clips were needed on average. The median procedure time was $18 \mathrm{~min}$ (range 5-49 $\mathrm{min}$ ). The complication rate was $0 \%(25 / 25)$. The clinical success rates on days 1
\end{abstract}


and 5 were $100 \%(19 / 19)$ and $100 \%(9 / 9)$, respectively. No patients presented stenosis as a late complication at 2 months after the procedure (0/25). Conclusion: The SCCT could be applied in the treatment of artificial ulcers in several parts of the Gl tract with a high clinical success rate and no complications. The SCCT appears to be a good option for closing artificial mucosal ulcers.

(C) 2019 The Author(s)

Published by S. Karger AG, Basel

\section{Introduction}

It is generally accepted that endoscopic procedures, including endoscopic mucosal resection (EMR) and endoscopic submucosal dissection (ESD) are effective for the treatment of early-stage gastrointestinal (GI) neoplastic lesions [1-3]. It is important to appropriately manage patients with procedure-related artificial mucosal ulcers or, if they occur, procedurerelated complications such as bleeding and perforation [4-6]. Perforation is one of the most severe complications since it not only causes high fever and abdominal pain but also sometimes requires emergent surgical treatment $[7,8]$. Recently, some reports have described the efficacy of closure for artificial ulcer after endoscopic treatment, such as ESD or EMR [9-12]. To date, artificial mucosal ulcers are most commonly closed using clip techniques, in order to prevent delayed perforation [9]. However, we sometimes encounter cases in which complete closure of an artificial mucosal ulcer is difficult due to its large size, complex shape, or location. Harada et al. [12] have reported the rate of complete clip closure after colonic ESD to be approximately $59 \%$, which is not satisfactory.

An open biopsy, such as a mucosal incision-assisted biopsy (MIAB), for subepithelial lesions (SELs) requires closing the ulcer in order to prevent delayed bleeding and perforation after the procedure. This is because an MIAB for SELs, which arise from the muscular layer or submucosal layer with rich vascularization, carries a potential risk of delayed perforation or delayed bleeding after the procedure. We also sometimes encounter difficult cases in which complete closure of an artificial mucosal ulcer is difficult even for small ulcers, as the character of ulcers at SELs created by an MIAB is quite different from that of ulcers at superficial tumors created by ESD. The mucosa at SELs is stretched strongly by the lesion, and closing the ulcer using only clips without pinching the SEL is difficult.

Many closure techniques, including over the scope clip (OTSC) techniques [13, 14], and combination clip and loop techniques [15-17], were developed for cases in which the closure of artificial mucosal ulcers is difficult to achieve by simple clip methods. These techniques have been shown to be useful; however, they require the use of special devices or an uncommon endoscope equipped with two working channels, which prevents these methods from being widely applied in clinical practice. Thus, a new method has been desired.

We recently developed a modified single-channel endoscopic closure technique (SCCT) using a conventional endoloop and clips. The purpose of this study is to evaluate the efficacy and safety of the SCCT.

\section{Methods}

Study Population and Protocols

Between December 2017 and September 2018, a total of 96 consecutive patients who underwent ESD for GI superficial tumors, EMR for duodenum superficial tumor, or a MIAB for SELs were enrolled in this study (ESD 79 cases, EMR for duodenum 1 case, MIAB 15 cases). All procedures were performed at Kyushu University. The patients in whom postprocedure ulcer closure was attempted endoscopically using SCCT were analyzed. In particular, in the ESD group, 11 patients in whom the ulcer size was over $40 \mathrm{~mm}$, which is the maximum 


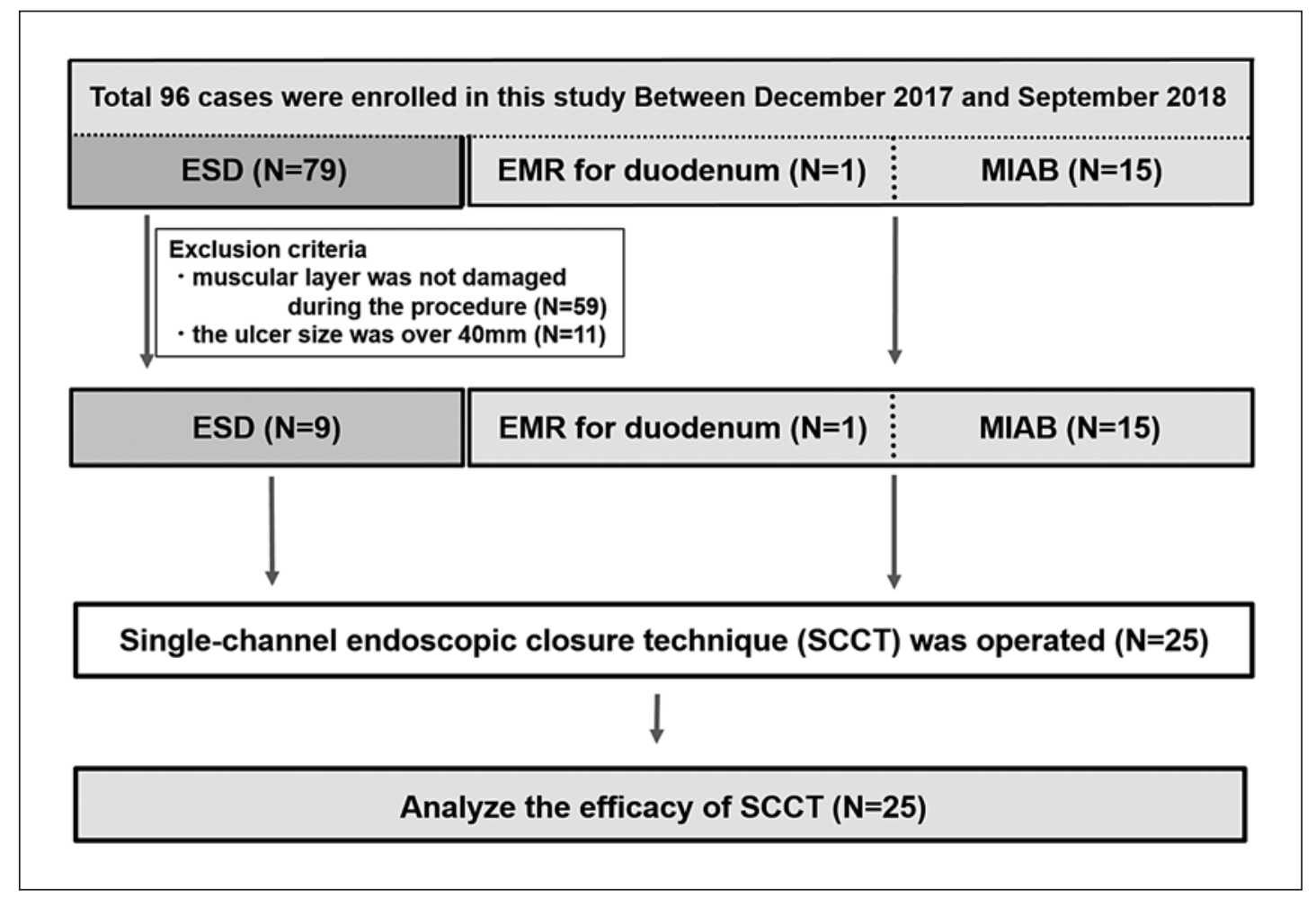

Fig. 1. Flowchart of patients with lesions enrolled in this study.

size of the endoloop and the largest size indicated for SCCT, did not undergo endoscopic closure and were therefore excluded from this study. In addition, 59 patients in whom the muscular layer was not damaged during the procedure and who were expected to have a low risk of delayed perforation were also outside of the indication for endoscopic closure and were thus excluded from the study. All endoscopic closures for ulcers $<40 \mathrm{~mm}$ were performed using the SCCT technique. As a result, 25 consecutive patients (male, $n=12$, female, $n=13$ ) who underwent SCCT for artificial mucosal ulcers after ESD, EMR for GI tumors, or a MIAB for SELs were analyzed retrospectively in the present study (Fig. 1). The steps of ESD, and MIAB were followed according to those described in a previous report $[18,19]$. Written informed consent for the procedure was obtained from all patients. To evaluate the efficacy and safety of this technique, we evaluated the technical success rate, number of clips for closure, procedure time, and complication rate on the day of procedure, the clinical success rate on the next day (day 1 ) and the 5 th day (day 5) after the procedure, and the incidence of severe stenosis of the GI tract after ulcer healing at 2 months after the procedure.

Technical success was defined as complete closure of the ulcer and the procedure time was calculated as the time from the insertion of the endoloop into the endoscope to the completion of loop closure. This time was calculated retrospectively, based on the time on the endoscopic images. Clinical success was defined by the X-ray findings that the clips remained in the proper position in the stomach without any procedurerelated symptoms (Fig. 2). Severe stenosis was defined by a complaint (from the patient) of stenosis-related symptoms or relevant air-fluid findings on abdominal X-ray suggesting severe bowel stenosis or obstruction. To determine whether severe stenosis occurred after discharge, a follow-up clinical examination was performed at 2 months after the procedure, and late-onset complications were recorded. This study was approved by the institutional ethics committee.

\section{Preparation of SCCT}

Preparation for the SCCT was performed as follows (Fig. 3). First, the stopper was moved slightly forward to the top of the loop (Endoloop; Olympus Corporation, Tokyo, Japan) (Fig. 3a). Second, after releasing the loop once, the loop tail was then sufficiently widened by hand (Fig. 3b, c). Third, the loop was placed directly into the outer sheath without fixing the ligation device (Fig. 3d). 
Gastro

Intestinal

Tumors

Fig. 2. Representative abdominal $\mathrm{X}$-ray image, which shows clips remaining in the proper position in the stomach.

\begin{tabular}{l|l}
\hline Gastrointest Tumors 2020;7:21-29 \\
\hline DOI: 10.1159/000503994 & $\begin{array}{l}\text { @ } 2019 \text { The Author(s). Published by S. Karger AG, Basel } \\
\text { www.karger.com/gat }\end{array}$ \\
\hline
\end{tabular}

Minoda et al.: The Efficacy of a Promising Single-Channel Endoscopic Closure Technique for Artificial Ulcers

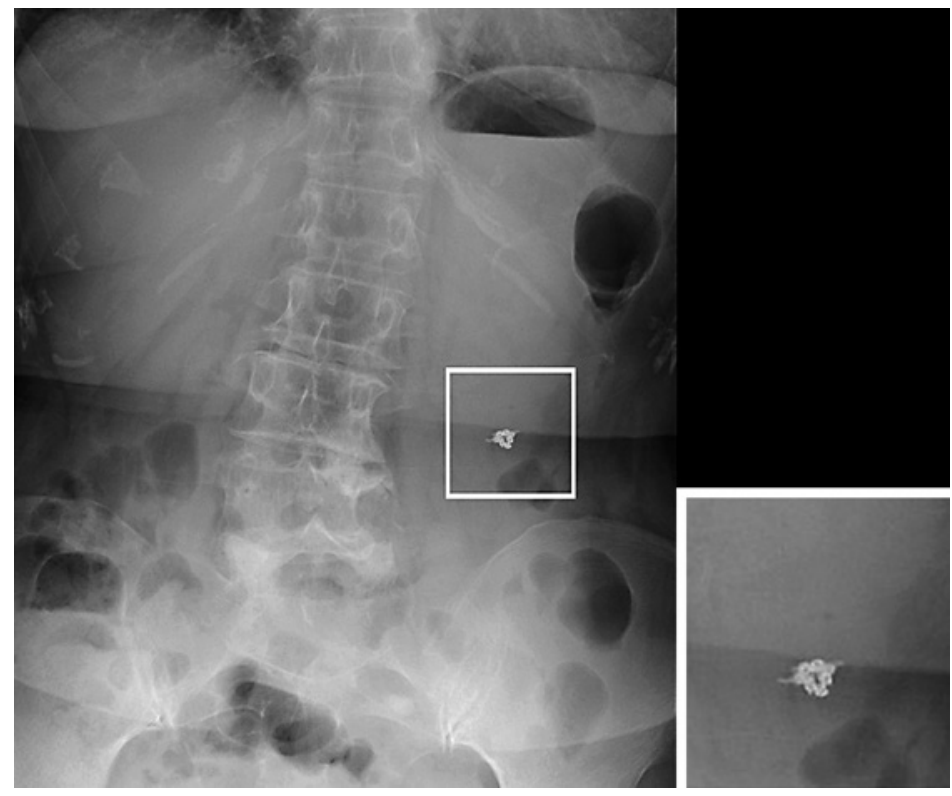

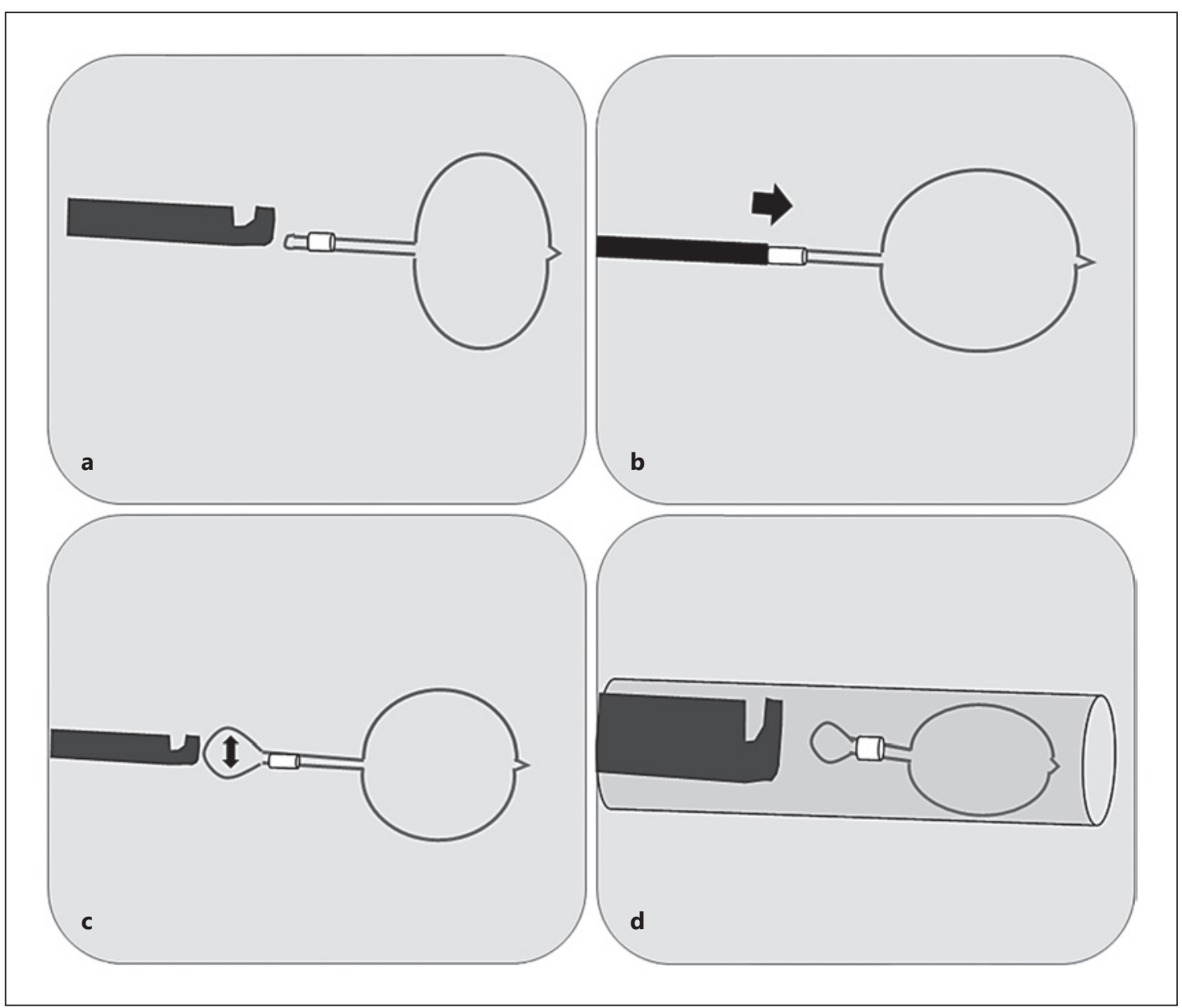

Fig. 3. A schematic illustration depicting the preparations for the SCCT. $\mathbf{a}, \mathbf{b}$ Moving the stopper forward to the top of the loop. $\mathbf{c}$ Releasing the loop from the ligation device and widening the loop tail sufficiently. $\mathbf{d}$ Putting the loop directly into the outer sheath. The modified loop is stored in the outer sheath. 


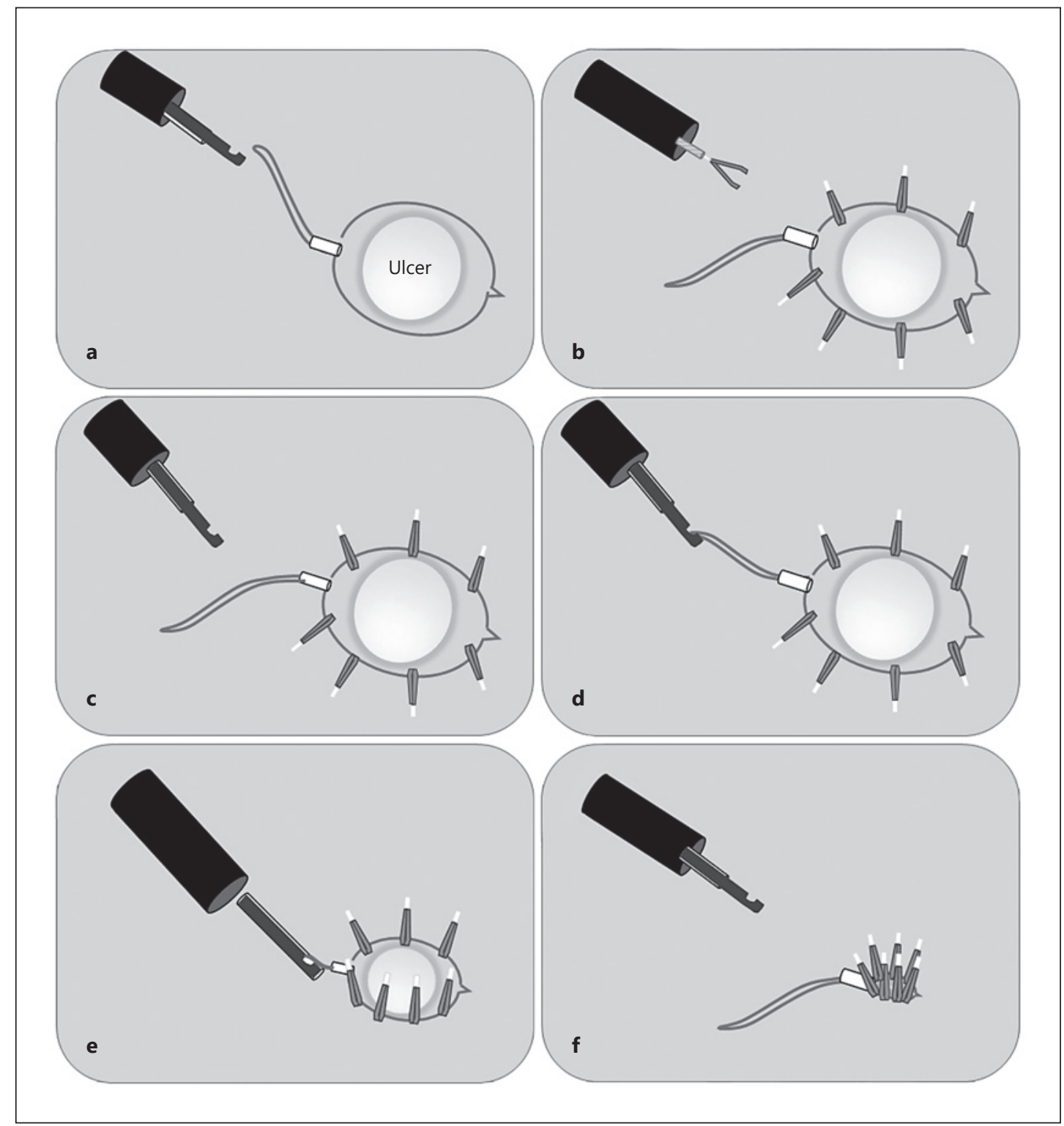

Fig. 4. A schematic illustration of the SCCT. a Dropping the tail of the loop. $\mathbf{b}$ Fixing the loop to the mucosa surrounding the ulcer by clips. c, $\mathbf{d}$ Catching the tail of the loop with the ligation device. $\mathbf{e}, \mathbf{f}$ Closing the loop tightly.

\section{Steps of SCCT}

The SCCT was then performed using a single channel endoscope (GIF-Q260J, PCF-Q260JI, PCF-Q260AZI; Olympus) as follows (Fig. 3, 4). First, the endoloop was dropped around the ulcer (Fig. 4a). Second, it was fixed using clips (ZEOCLIP ${ }^{\circledR}$, Zeon Medical, Japan; EZ clip ${ }^{\circledR}$, Olympus) (Fig. 4b). Then, the endoloop was tightened using a closure device in the GI tract (Fig. 4c, d), and the endoloop was closed (Fig. 4e, f).

\section{Tips for SCCT}

The main point to bear in mind when performing the SCCT is to align the direction of the loop tail with that of the scope. To make this alignment, the scope should be pulled back a little after inserting the ligation device into the hole of loop tail. Pulling back the scope causes the direction of the scope and the loop to align. After that, the ligation device should be closed slowly. 


\section{Gastro \\ Intestinal \\ Tumors}

Table 1. Characteristics of the patients enrolled in this study

\begin{tabular}{l|l}
\hline Gastrointest Tumors 2020;7:21-29 \\
\hline DOI: 10.1159/000503994 & $\begin{array}{l}\text { @ 2019 The Author(s). Published by S. Karger AG, Basel } \\
\text { www.karger.com/gat }\end{array}$ \\
\hline
\end{tabular}

Minoda et al.: The Efficacy of a Promising Single-Channel Endoscopic Closure Technique for Artificial Ulcers

\begin{tabular}{ll} 
Age, years & 65 (range $36-81)$ \\
Males/females & $12 / 13$ \\
Ulcer location, $n$ cases $^{\mathrm{a}}$ & \\
$\quad$ Stomach (upper) & $8(1 / 7 / 0)$ \\
$\quad$ Stomach (middle) & $6(0 / 6 / 0)$ \\
Stomach (lower) & $5(3 / 2 / 0)$ \\
Duodenum & $1(0 / 0 / 1)$ \\
Colon (ascending) & $1(1 / 0 / 0)$ \\
Colon (transverse) & $2(2 / 0 / 0)$ \\
Colon (descending) & $1(1 / 0 / 0)$ \\
Rectum & $1(1 / 0 / 0)$ \\
Type of treatment, $n$ cases & \\
ESD & 9 \\
MIAB & 15 \\
EMR & 1 \\
Median diameter of ulcer, mm & 20 (range $10-40)$ \\
ESD & 26 (range $15-40)$ \\
MIAB & 15 (range $10-40)$ \\
EMR & 10 \\
\hline
\end{tabular}

${ }^{\mathrm{a}}$ Total (ESD/MIAB/EMR).
Table 2. Efficacy and safety of the modified single-channel endoscopic closure technique
Technical success rate (complete closure)

Procedure time, min

Number of clips

Complication rate

Clinical success on day 1

Clinical success on day 5

Severe stenosis 
release in order to make it work. Most ligation devices are not appropriate for this procedure as it stands, since the shape of the loop tail is too straight and narrow to hold in the final step. In many countries, the conventional endoloop has a straight tail. We have improved this point and developed our method. It is important to address two points in order to keep the tail of the endoloop round when preparing to perform the SCCT. First, the tail of the endoloop should be widened to make the loop tail easier to catch in the final step. A round and wide loop tail is suitable for the SCCT. Second, the loop should be placed directly into the outer sheath without fixing the tail of the loop to the ligation device. Although the preliminary trials of the SCCT using a conventional straight-tail endoloop often failed, the adoption of the two abovementioned points enabled us to perform the SCCT more easily. One strength of this method is that SCCT can be applied to almost all types of ligation loops. The loop tail needs to be large and wide enough to hold during live endoscopy.

Because the shape of the endoloop is changed and deformed to some degree, it has not fully been determined whether this SCCT method can be applied in other sites in the GI tract, including the stomach, duodenum, and colon. Thus, the present study was performed. In the present study, artificial ulcers could be successfully managed by the SCCT regardless of the location. The locations in which the technique was applied included the upper stomach, middle stomach, lower stomach, duodenum, ascending colon, transverse colon, and rectum.

In the present study, we also showed that the technical success rate of the SCCT was $100 \%$, while the incomplete closure rate and complication rate were both $0 \%$. Using this technique, all artificial ulcers could be closed completely, easily, and safely. Moreover, the clinical success rate on day 5 was also $100 \%$. In general, delayed complications such as perforation or bleeding after endoscopic treatment occur within several days after the procedure. Our data suggest that SCCT may reduce the occurrence of delayed perforation or the bleeding risk of artificial ulcers.

In the present study, the median procedure time was $18 \mathrm{~min}$. We consider it to be acceptable. It is more time consuming to use the special devices required for OTSC, where the endoscope is pulled out once, then the OTSC device is set up and the endoscope is inserted to access the lesion. The SCCT enables us to perform the closure of the mucosal ulcers immediately after the resection procedure without the reinsertion of the scope. The SCCT can be performed in general clinical practice, since it does not require any special devices or endoscopes.

The performance of EFTR for submucosal tumors has been reported and has gradually spread throughout the world [23-27]. The techniques of EFTR have advanced with methods for closing wall defect. Many techniques to close the wall defect require special devices such as OTSCs and suturing devices. However, some studies have reported that a combination method using endoloops and conventional clips is sufficient to close mucosal defect after EFTR $[20,28]$. Thus, the SCCT would be useful not only for ESD but also for EFTR.

Another advantage of this technique is its cost-effectiveness. SCCT required approximately eight clips and one endoloop, which comes to an approximate cost of USD 80-100 (USD 8-9 for 1 clip $\times 8$ clips and USD 20-25 for 1 endoloop). This cost is markedly lower than that of OTSCs (USD 700-800 for a single OTSC).

The present study has several limitations. The first limitation is selection bias. In this study, there were many cases in which several different procedures, such as ESD, EMR, or MIAB, were used. In addition, the concerned organs in the enrolled patients varied (stomach, duodenum, colon, and rectum). Second, this was a retrospective study. Further studies, including multicenter prospective studies, will be required to establish these methods. Third, we did not evaluate the clinical success or stenosis endoscopically, although on the abdominal $\mathrm{X}$-ray images the clips remained in the proper position in all cases on the first days. Since $\mathrm{X}$-ray images are two-dimensional evaluations, our findings do not show the complete closure 
Minoda et al.: The Efficacy of a Promising Single-Channel Endoscopic Closure

Technique for Artificial Ulcers

of the ulcers. This remains one of the limitations of this study. Finally, the examined ulcer size was set at $<40 \mathrm{~mm}$ because the maximum size of the endoloop is $40 \mathrm{~mm}$.

In conclusion, the SCCT was applicable in the treatment of artificial ulcers in several parts of the GI tract and showed a high clinical success rate with no complications. The SCCT appears to be a good option for closing artificial mucosal ulcers.

\section{Statement of Ethics}

The study was conducted in accordance with the Declaration of Helsinki, and the study protocol was approved by our Institute's Ethical Committee. Written informed consent for the procedure was obtained from all patients.

\section{Disclosure Statement}

The authors have no conflicts of interest to declare.

\section{Funding Sources}

None.

\section{Author Contributions}

Yosuke Minoda designed the study, analyzed the data, and wrote the manuscript. Haruei Ogino edited and reviewed the manuscript. Eikichi Ihara collected data and critically reviewed the manuscript. All other authors have contributed to the collection of the data.

\section{References}

1 Thorlacius H, Rönnow CF, Toth E. European experience of colorectal endoscopic submucosal dissection: a systematic review of clinical efficacy and safety. Acta Oncol. 2019;58(sup1):S10-4.

2 Park YM, Cho E, Kang HY, Kim JM. The effectiveness and safety of endoscopic submucosal dissection compared with endoscopic mucosal resection for early gastric cancer: a systematic review and metaanalysis. Surg Endosc. 2011 Aug;25(8):2666-77.

3 Ferlitsch M, Moss A, Hassan C, Bhandari P, Dumonceau JM, Paspatis G, et al. Colorectal polypectomy and endoscopic mucosal resection (EMR): European Society of Gastrointestinal Endoscopy (ESGE) Clinical Guideline. Endoscopy. 2017 Mar;49(3):270-97.

4 Goto O, Fujishiro M, Oda I, Kakushima N, Yamamoto Y, Tsuji Y, et al. A multicenter survey of the management after gastric endoscopic submucosal dissection related to postoperative bleeding. Dig Dis Sci. 2012 Feb;57(2): 435-9.

5 Chiba H, Ohata K, Tachikawa J, Arimoto J, Ashikari K, Kuwabara H, et al. Delayed Bleeding After Colorectal Endoscopic Submucosal Dissection: When Is Emergency Colonoscopy Needed? Dig Dis Sci. 2019 Mar;64(3): 880-887.

6 Harada H, Suehiro S, Murakami D, Nakahara R, Nagasaka T, Ujihara T, et al. Feasibility of gastric endoscopic submucosal dissection with continuous low-dose aspirin for patients receiving dual antiplatelet therapy. World J Gastroenterol. 2019 Jan;25(4):457-68.

7 Fujihara S, Mori H, Kobara H, Nishiyama N, Matsunaga T, Ayaki M, et al. Management of a large mucosal defect after duodenal endoscopic resection. World J Gastroenterol. 2016 Aug;22(29):6595-609.

8 Inoue T, Uedo N, Yamashina T, Yamamoto S, Hanaoka N, Takeuchi Y, et al. Delayed perforation: a hazardous complication of endoscopic resection for non-ampullary duodenal neoplasm. Dig Endosc. 2014 Mar;26(2): 220-7.

9 Osada T, Sakamoto N, Ritsuno H, Murakami T, Ueyama H, Matsumoto K, et al. Closure with clips to accelerate healing of mucosal defects caused by colorectal endoscopic submucosal dissection. Surg Endosc. 2016 Oct; 30(10):4438-44. 
Minoda et al.: The Efficacy of a Promising Single-Channel Endoscopic Closure

Technique for Artificial Ulcers

10 Ogiyama H, Tsutsui S, Murayama Y, Maeda S, Satake S, Nasu A, et al. Prophylactic clip closure may reduce the risk of delayed bleeding after colorectal endoscopic submucosal dissection. Endosc Int Open. 2018 May; 6(5):E582-8.

11 Kato M, Ochiai Y, Fukuhara S, Maehata T, Sasaki M, Kiguchi Y, et al. Clinical impact of closure of the mucosal defect after duodenal endoscopic submucosal dissection. Gastrointest Endosc. 2019 Jan;89(1):87-93.

12 Harada H, Suehiro S, Murakami D, Nakahara R, Ujihara T, Shimizu T, et al. Clinical impact of prophylactic clip closure of mucosal defects after colorectal endoscopic submucosal dissection. Endosc Int Open. 2017 Dec; 5(12):E1165-71.

13 Tashima T, Ohata K, Sakai E, Misumi Y, Takita M, Minato Y, et al. Efficacy of an over-the-scope clip for preventing adverse events after duodenal endoscopic submucosal dissection: a prospective interventional study. Endoscopy. 2018 May;50(5):487-96.

14 Kobara H, Mori H, Nishiyama N, Fujihara S, Okano K, Suzuki Y, et al. Over-the-scope clip system: A review of 1517 cases over 9 years. J Gastroenterol Hepatol. 2019 Jan;34(1):22-30.

15 Abe S, Oda I, Mori G, Nonaka S, Suzuki H, Yoshianaga S, et al. Complete endoscopic closure of a large gastric defect with endoloop and endoclips after complex endoscopic submucosal dissection. Endoscopy. 2015;47 Suppl 1 UCTN:E374-5.

16 Wang J, Wang X, Liu L, Zhao L, Wang M, Liu Z, et al. Endoscopic closure of a colonic defect using a novel endoloop system via a single-channel endoscope. Endoscopy. 2016;48(S 01 Suppl 1):E142-3.

17 Wang J, Zhao L, Wang X, Liu L, Wang M, Fan Z. A novel endoloop system for closure of colonic mucosal defects through a single-channel colonoscope. Endoscopy. 2017 Aug;49(8):803-7.

18 Akahoshi K, Akahane H, Motomura Y, Kubokawa M, Itaba S, Komori K, et al. A new approach: endoscopic submucosal dissection using the Clutch Cutter ${ }^{\circledR}$ for early stage digestive tract tumors. Digestion. 2012;85(2): 80-4.

19 Ihara E, Matsuzaka H, Honda K, Hata Y, Sumida Y, Akiho H, et al. Mucosal-incision assisted biopsy for suspected gastric gastrointestinal stromal tumors. World J Gastrointest Endosc. 2013 Apr;5(4):191-6.

20 Meng FS, Zhang ZH, Hong YY, Li DJ, Lin JQ, Chen X, et al. Comparison of endoscopic submucosal dissection and surgery for the treatment of gastric submucosal tumors originating from the muscularis propria layer: a single-center study (with video). Surg Endosc. 2016 Nov;30(11):5099-107.

21 Matsuda T, Fujii T, Emura F, Kozu T, Saito Y, Ikematsu H, et al. Complete closure of a large defect after EMR of a lateral spreading colorectal tumor when using a two-channel colonoscope. Gastrointest Endosc. 2004 Nov; 60(5):836-8.

22 Huang S, Zhu S. Closure of duodenal ulcer perforation using a novel endoloop device with a single-channel gastroscope after failed laparoscopic repair. Endoscopy. 2017 Feb;49 S 01:E31-2.

23 Andrisani G, Soriani P, Manno M, Pizzicannella M, Pugliese F, Mutignani M, et al. Colo-rectal endoscopic fullthickness resection (EFTR) with the over-the-scope device $\left(\right.$ FTRD $^{\circledR}$ ): A multicenter Italian experience. Dig Liver Dis. 2019 Mar;51(3):375-381.

24 Chiu PW, Yip HC, Teoh AY, Wong VW, Chan SM, Wong SK, et al. Per oral endoscopic tumor (POET) resection for treatment of upper gastrointestinal subepithelial tumors. Surg Endosc. 2019 Apr;33(4):1326-33.

25 Kitakata H, Itoh T, Kinami S, Kawaura K, Hamada K, Azukisawa S, et al. Sealed endoscopic full-thickness resection for gastric cancer: a pilot study in an ex vivo and in vivo porcine model. Endosc Int Open. 2019 Jan; 7(1):E36-42.

26 Kuellmer A, Mueller J, Caca K, Aepli P, Albers D, Schumacher B, et al.; FTRD study group. Endoscopic fullthickness resection for early colorectal cancer. Gastrointest Endosc. 2019 Jun;89(6):1180-1189.e1.

27 Li J, Meng Y, Ye S, Wang P, Liu F. Usefulness of the thread-traction method in endoscopic full-thickness resection for gastric submucosal tumor: a comparative study. Surg Endosc. 2019 Sep;33(9):2880-2885.

28 Shi D, Li R, Chen W, Zhang D, Zhang L, Guo R, et al. Application of novel endoloops to close the defects resulted from endoscopic full-thickness resection with single-channel gastroscope: a multicenter study. Surg Endosc. 2017 Feb;31(2):837-42. 